\title{
Letter to editor: "Vaginal Er:YAG laser application in the menopausal ewe model: a randomised estrogen and sham-controlled trial "
}

\author{
HSU CHENG YANG ${ }^{1}$, Ching-Hu $\mathrm{Wu}^{2}$, and Cheng-Yu Long ${ }^{3}$ \\ ${ }^{1}$ Kaohsiung Medical University Hospital \\ ${ }^{2}$ Affiliation not available \\ ${ }^{3}$ Kaohsiung Municipal Siaogang Hospital
}

July 8, 2021

Letter to editor: "Vaginal Er:YAG laser application in the menopausal ewe model: a randomised estrogen and sham-controlled trial "

Cheng-Yang $\mathrm{Hsu}^{1}$, Ching-Hu Wu ${ }^{1}$, Cheng-Yu Long ${ }^{1,2}$

${ }^{1}$ Department of Obstetrics and Gynecology, Kaohsiung Medical University Hospital, Kaohsiung Medical University, Kaohsiung, Taiwan

${ }^{2}$ Department of Obstetrics and Gynecology, Kaohsiung Municipal Siaogang Hospital, Kaohsiung Medical University, Kaohsiung, Taiwan

Running head: Two prospective for this promising experiment

Words Count: 315

Corresponding Author:

Dr. Cheng-Yu Long, MD, PhD, Department of Obstetrics and Gynaecology, Kaohsiung Medical University Hospital, Kaohsiung Medical University, 100, Shih-Chuan 1st Rd, Kaohsiung 80708, Taiwan

E-mail: urolong@yahoo.com.tw

Dear Editor-in Chief:

We read with great interest with recent publication in BJOG by Mackova et al. (1) This study describe effects of non-ablative erbium-doped: yttrium-aluminium-garnet (Er:YAG) laser on vaginal atrophy induced by iatrogenic menopause in the ewe. The ewes were randomized to three groups: vaginal Er:YAG laser application, estrogen replacement, and sham groups.

In the estrogen replacement group of ewes, an estrogen implant was inserted under the skin in the inguinal region. The result showed increase in epithelia thickness in vaginal biopsies and it was significantly higher compared with the laser application and sham groups. In all groups, the lamina propria did not showed any significant differences. Also the autopsy showed the uterus of estrogen-exposed ewes weighted more.

We would like to humbly comment the vaginal estrogen use and laser application in genitourinary syndrome of menopause.

The vaginal estrogen application was given twice a week with the cream in currently practice of female who suffered from genitourinary syndrome of menopause (GSM). Firstly, the vaginal estrogen application was 
given twice a week with the cream in clinical practice of female who suffered from GSM. The administration showed improvement of the symptoms while no significant change in serum estrogen level. (2) The estrogen implant in the ewe experiment continues to release estrogen and caused sustained estrogen effect in the ewe and caused gaining weight of uterus, which was very different with our clinical practice of intermittent vaginal cream use.

Second, the application in this study is Er:YAG laser, with the the lesser wave length: $2940 \mathrm{~nm}$. While the $\mathrm{CO}_{2}$ laser had much longer wave length $10600 \mathrm{~nm}$ and had deeper effect in not only vaginal epithelium but also lamina propia. (3) Therefore the vascularization effects of lamina propia may be obvious noticeably.

We thought these 2 flaws could be revised to make the ewe experiment more accurate and similar to current management in women with GSM.

References

1. Mackova KA-OX, Mazzer AM, Mori Da Cunha MA-O, Hajkova Hympanova LA-O, Urbankova IA-O, Kastelein AA-O, et al. Vaginal Er:YAG laser application in the menopausal ewe model: a randomised estrogen and sham-controlled trial. BJOG . 2021 May;128(6):1087-1096.

2. Long CY, Liu CM, Hsu SC, Wu CH, Wang CL, Tsai EM. A randomized comparative study of the effects of oral and topical estrogen therapy on the vaginal vascularization and sexual function in hysterectomized postmenopausal women. Menopause. Sep-Oct 2006;13(5):737-43.

3. Bhide AA, Khullar V, Swift S, Digesu GA. The use of laser in urogynaecology. Int Urogynecol J . 2019 May;30(5):683-692. 